Editorial

\title{
Technology behaviors in education innovation
}

Change and improvement are two keywords embedded in innovation in general (OECD, 2005) and, in particular, in teaching and learning innovation (Miles, 1964). Based on those two keywords, educational innovation could be defined as "the application of one idea that produces a planned change in educational processes, services, or products, then leading to an improvement in learning goals". The role of the computer in educational innovation is seen as a facilitating tool, as both educational innovation and computation address the same topic, i.e. "Knowledge".

The computer's capability to manage information makes it an ideal tool to potentiate different implementations in teaching and learning contexts. The several distinct ways teachers and students interact are oriented by teaching methods. This means the computer may be used to: improve existing methods for teacherstudents interaction, e.g. traditional lectures; enable alternative methods that are difficult to apply under current conditions, e.g. personalized learning; create new methods, e.g. flipped teaching; or, in addition, analyse data generated from teacher-students interactions and help in the learning improvement decision-making process (Fidalgo-Blanco, Sein-Echaluce, García-Peñalvo, \& Conde, 2015).

Although teaching staff has been innovating for decades, presently it is the knowledge society that offers more opportunities for innovation. Computers, information and communication technologies, cloud computing, new trends in cooperative work, and the widespread dissemination and use of knowledge are generating a large number and variety of technology-enhanced educational tools, which allow teachers to do new experiments, convert an improvement idea into an effective improvement process, and, definitely, to innovate the learning process.

From a training perspective, all educational systems share the same goals and aims. They also share the same problems, for instance school failure and the promotion of student-centred active learning practices. The idea and the conviction that educational innovation helps to improve learning goals and students' learning skills are also shared among all stakeholders.

For these reasons, the techniques, technologies and strategies used in innovative educational experiences are universally transferable and exportable, irrespectively of the curricula and academic scope in which they have been trialled.

This special issue includes 17 articles addressing different experiences of educational innovation that share a common goal, i.e. the use of technology to improve learning, while adopting different strategies to fulfil it. The following abstracts provide readers with a concise overview of this special issue contents.
Technological adaptation of the student to the educational density of the course. A case study: 3D Architectural Visualization David Fonseca (Ramon Llull University), Ernesto Redondo (Polytechnic University of Catalonia), Francesc Valls (Polytechnic University of Catalonia) and Sergi Villagrasa (Ramon Llull University). This article proposes to assess the degree of students' adaptation to two types of courses on applications of architectural spatial representation. The authors consider two experiences with different number (5 and 20) of applications focused on the three-dimensional architectural (3D) representation that are offered to students and that promote active learning. The quantitative and qualitative data studies confirm that a high number of applications can stress the student, and with a smaller number of applications and more time for explanations a greater effectiveness in the learning process is obtained, as well as increasing the students' final motivation and satisfaction.

Learning with mobile technologies - students' behaviour. Laura Briz-Ponce (University of Salamanca), Anabela Pereira (University of Aveiro), Lina Carvalho (University of Coimbra), Juan Antonio Juanes-Méndez (University of Salamanca) and Francisco José García-Peñalvo (University of Salamanca) investigates on the different factors that could influence in students' behavior to use mobile technologies for learning. Data were obtained from health grades students and the results show a strong attitude towards using mobile technology for learning and willingness to recommend it.

Improving the expressiveness of black-box models for predicting student performance. Carlos J. Villagrá-Arnedo (University of Alicante), Francisco J. Gallego-Durán (University of Alicante), Faraón Llorens-Largo (University of Alicante), Patricia Compañ-Rosique (University of Alicante), Rosana Satorre-Cuerda (University of Alicante) and Rafael Molina-Carmona (University of Alicante). This article proposes a more expressive interpretation of black-box systems for predicting student performance which are based in a set of features useful for guiding the learning process. It includes experiment design aspects such as: defining the data to be collected, the algorithm to be used for the classification, the number and range of the classes, the output to be obtained, and so on. Besides, a set of graphical tools has been proposed to provide rich information of several types, which allows the detection of trends and behavioral patterns. A set of tips about how to design a prediction system and the representation of the output information is also provided.

Students' Perceptions and Attitudes towards asynchronous technological tools in Blended-Learning training to Improve Grammatical Competence in English as a Second Language. Ana $\mathrm{M}^{\mathrm{a}}$ PintoLlorente (Pontifical University of Salamanca), $\mathrm{M}^{\mathrm{a}}$ Cruz SánchezGómez (University of Salamanca), Francisco José García-Peñalvo 
(University of Salamanca) and Sonia Casillas-Martín (University of Salamanca). This article explores students' perception towards the effectiveness of certain asynchronous tools in blended learning (podcast, videocast, online tests, online glossary and forums) to acquire grammatical competence in English as a second language. Between the positive perceptions included by participants the following can be mentioned: a greater autonomy to set and organize their own learning, natural opportunities to access authentic materials and practice target language, support for collaborative and independent learning, motivational technique to maintain their interest through the e-activities or benefits of the e-activities to carry out a continuous assessment from tutors and students' selfassessment.

MLearning and pre-service teachers: An assessment of the behavioral intention using an expanded TAM model. José Carlos SánchezPrieto (University of Salamanca), Susana Olmos-Migueláñez (University of Salamanca) and Francisco J. García-Peñalvo (University of Salamanca). This article presents the results of a study on the behavioral intention of using mobile devices within the future teaching practice of pre-service primary education teachers. The authors indicate students perceive mobile devices as a familiar tool in their daily lives, although they have trouble picturing their use as a didactic tool. The analysis suggested that the stronger relationships were those established between perceived usefulness and behavioral intention, perceived ease of use and perceived usefulness, and self-efficacy and perceived ease of use. The authors suggest training actions should be centred in the diverse functionalities and didactic uses of these devices in real working contexts through practical activities.

Work Engagement and its Antecedents and Consequences: A Case of Lecturers Teaching Synchronous Distance Education Courses. IShuo Chen (Wuhan University). This article investigates the work engagement of lecturers teaching synchronous distance education courses and its antecedents (i.e. job demands/job resources) and consequences (i.e. lecturers' intention to continue to teach synchronous distance education courses). The author proves that the job demands negatively predict lecturers' work engagement, whereas job resources positively predict it and that lecturers' work engagement positively predicts their intention to continue to teach synchronous distance education courses and fully mediates the relationship between job demands and job resources and lecturers' intention to continue to teach synchronous distance education courses.

Collaborative learning and mobile devices: an educational experience in Primary Education. Ana Iglesias Rodríguez (University of Salamanca), Blanca García Riaza (University of Salamanca) and María Cruz Sánchez Gómez (University of Salamanca). This article is focused on the design, implementation and evaluation of a collaborative experience with ICT involving students of Primary Education in a Spanish school. The sample was made up by teachers, tutors, teacher ICT support and students. The results confirm that ICT provide tools and channels that have multiplied the possibilities to carry out collaborative projects, providing quality assurance to share and communicate, and, at the same time, confronts the dominant methodology based more on individual learning where the teacher continues to exercise the role of sole transmitter of knowledge.

Which cognitive abilities underlie computational thinking? Criterion validity of the Computational Thinking Test. Marcos RománGonzález (Universidad Nacional de Educación a Distancia), JuanCarlos Pérez-González (Universidad Nacional de Educación a Distancia) and Carmen Jiménez-Fernández (Universidad Nacional de Educación a Distancia). This article presents computational thinking as a set of problem-solving skills that must be acquired by the new generations of students to thrive in a digital world full of objects driven by software. The authors provide a new instrument for computational thinking measurement and additionally give evidence of the nature of computational thinking through its associations with key related psychological constructs such as: spatial ability, reasoning ability and problem-solving ability.

Investigating the effect of realistic projects on students' motivation, the case of Human-Computer interaction course. Jaime UrquizaFuentes (Universidad Rey Juan Carlos) and Maximiliano ParedesVelasco (Universidad Rey Juan Carlos). This article presents a study of students' motivation in methodologies which include realistic projects based on collaborative work, long term duration and multidisciplinary design interacting with real end-users. The authors show that, independently from the end-user recruitment, students involved in realistic projects are significantly more motivated than students involved in the general approach.

Supporting Technology-Enhanced Inquiry through Metacognitive and Cognitive Prompts: Sequential Analysis of Metacognitive Actions in Response to Mixed Prompts. Ying-Shao Hsu (National Taiwan Normal University), Chia-Yu Wang (National Chiao Tung University) and Wen-Xin Zhang (National Taiwan Normal University). This study develops and examines two types of support (cognitive and metacognitive) for inquiry practices in computer-based learning environments. The authors show that mixture of cognitive and metacognitive prompts helped the students clarifying the learning tasks, becoming aware of their learning difficulties, and selecting an appropriate strategy for ensuring the quality of their inquiry performance.

Micro Flip Teaching - An Innovative Model to Promote the Active Involvement of Students. Angel Fidalgo-Blanco (Technical University of Madrid), Margarita Martinez-Nuñez (Technical University of Madrid), Oriol Borrás-Gene (Technical University of Madrid) and Javier J. Sanchez-Medina (University of Las Palmas de Gran Canaria). This article measures the learning impact of micro flip teaching, a model that solves problems in the active participation of students in the classroom. The study shows the degree of students' involvement in their learning process through the creation of learning resources. The authors show a positive impact of micro flip teaching on the students learning and high perception of method usefulness, independently of the academic subject.

Developing A Culture of Commenting in a First-Year Seminar. Philip Kreniske (City University of New York). This article presents a study to examine how freshmen use a blog built within a seminar created to support their transition to college and to develop a culture of commenting. A narrative analysis allowed the author to measure the rates of specific evaluative devices in students' posts and to predict over $50 \%$ of the commenting variance.

Differences between prospective, existing, and former users of interactive whiteboards on external factors affecting their adoption, usage and abandonment. Boštjan Sumak (University of Maribor), Maja Pušnik (University of Maribor), Marjan Heričko (University of Maribor) and Andrej Šorgo (University of Maribor). This article shows the development and validation of an instrument to measure the users' perceptions about performance expectancy and effort expectancy during different technology adoption stages. In this case the technology considered is the interactive whiteboards. The authors show that user interface quality, personal innovativeness and perceived pedagogical impact are factors that affect teachers' perceptions in all adoption stages.

Using Gameplay Data to Examine Learning Behavior Patterns in a Serious Game. Jina Kang (The University of Texas at Austin), Min Liu (The University of Texas at Austin) and Wen Qu (University of Notre Dame). This study identifies students' learning behavior patterns of problem-solving and explore behavior patterns of different performing groups within a specific open-ended serious game. The proposed sequential pattern mining allows a better understanding 
of the learning pathway of students and problem-solving strategies, by considering different learning characteristics in a serious games context.

Enriching Programming Content Semantics: An Evaluation of Visual Analytics Approach. I-Han Hsiao (Arizona State University) and Yi-Ling Lin (National Sun Yat-Sen University). This article shows the design and study of a semantic indexing method via visual analytics, which indexes article-based programming problems to sets of concepts. It is shown that dynamic concept indexing provides teachers with immediate feedback on producing more balanced exams. The results show that the method allows making traces of learning analytics in semantic level and provides more personalized feedback for students, which is normally difficult in traditional classrooms.

Validation of indicators to implement an adaptive platform for MOOCs. Dolores Lerís (University of Zaragoza), María Luisa SeinEchaluce (University of Zaragoza), Miguel Hernández (Catholic University of Valencia) and Concepción Bueno (University of Zaragoza). This article shows that previous works consider personalized learning methodologies as the solution for learning difficulties in Massive Open Online Courses (MOOCs). The authors show the consistent scale of a construct of adaptivity for MOOCs whose indicators are based on learning self-regulation and cooperation. The study shows the most preferred adaptivity indicators for participants in MOOCs and the high demand of adaptivity characteristics if participants have no previous completion in MOOCs.

A proposed Paradigm for Smart Learning Environment Based on Semantic Web. Shimaa Ouf (Helwan University), Mahmoud Abd Ellatif (Helwan University), Shaimaa Salama (Helwan University) and Yehia Helmy (Helwan University). This article proposes and implements a framework for a smart e-learning ecosystem using ontology and semantic web rule language. It proposes a new direction which fosters the creation of four separate ontologies for the personalized full learning package which is composed of the learner model and all the learning process components (learning objects, learning activities and teaching methods).

The articles included in this special issue are a small example of the wide variety of studies, experiences and developments in which technologies are the support to innovation in education. In any case they cover methodologies (blended and distance learning, visual learning, collaborative learning, open learning, personalized learning, flip teaching, social learning), with the help of technologies (mobile devices, predicted devices, learning content management systems, learning analytics, visual analytics, gamification, semantic web) and bearing in mind cognitive and metacognitive aspects to the acquisition, improvement and evaluation of competences (visual, computational, collaborative, self-regulation). The join of those methodologies, technologies and competences compose the "technological ecosystems" which are a trending topic nowadays (García-Peñalvo et al., 2015). We hope they inspire new researches and studies focused in the aim of educational innovation, i.e. the improvement of learning process.

\section{Acknowledgements}

The guest editors are very thankful to Professor Robert Tennyson, Editor of Computers in Human Behavior, for the opportunity to organize this special issue, Asha Thayappan, CHB Journal Manager, Kavitha Narayanasamy, Publishing Content Specialist for $\mathrm{CHB}$, and the rest of the journal staff for their support, and to the reviewers who generously devoted time to provide feedback to the authors and ensured the quality of this special issue.

\section{References}

Fidalgo-Blanco, Á, Sein-Echaluce, M. L. García-Peñalvo, F. J. \& Conde, M.Á. (2015) Using Learning Analytics to improve teamwork assessment. Computers in $\mathrm{Hu}$ man Behavior, 47, 149-156. https://doi.org/10.1016/j.chb.2014.11.050.

García-Peñalvo, F. J., Hernández-García, Á., Conde, M.Á., Fidalgo-Blanco, Á., SeinEchaluce, M. L., Alier-Forment, M., et al. (2015). Enhancing education for the knowledge society era with learning ecosystems. In F. Garcia-Peñalvo, \& A. García-Holgado (Eds.), Open source solutions for knowledge management and technological ecosystems (pp. 1-24). Hershey, PA: IGI Global. http://dx.doi.org/ 10.4018/978-1-5225-0905-9.ch001.

Miles, M. B. (1964). Educational Innovation: Resources, strategies, and unanswered questions. American Behavioral Scientist, 7(6), 10-14. https://doi.org/10.1177/ 000276426400700604.

OECD. (2005). Oslo manual. Guidelines for collecting and interpreting innovation (3rd ed.). OECD Publishing https://doi.org/10.1787/9789264013100-en.

María Luisa Sein-Echaluce*

Department of Applied Mathematics, University of Zaragoza, School of Engineering and Architecture, María de Luna, 3, 50018 Zaragoza,

Spain

Ángel Fidalgo-Blanco

Department of Geological and Mining Engineering, Technical University of Madrid, School of Mining and Energy Engineering, Ríos Rosas, 21, 28003 Madrid, Spain E-mail address: angel.fidalgo@upm.es.

Gustavo Alves

Department of Electrical Engineering, Polytechnic of Porto, School of Engineering, Rua Dr. António Bernardino de Almeida, 431, 4200-072 Porto, Portugal

E-mail address: gca@isep.ipp.pt.

* Corresponding author. E-mail address: mlsein@unizar.es (M.L. Sein-Echaluce).

Available online $\mathrm{xxx}$ 